\title{
Hybrid steepest iterative algorithm for a hierarchical fixed point problem
}

\author{
Shamshad Husain * and Nisha Singh
}

${ }^{\text {*Correspondence: }}$

s_husain68@yahoo.com

Department of Applied

Mathematics, Aligarh Muslim

University, Aligarh, 202002, India

\begin{abstract}
The purpose of this work is to introduce and study an iterative method to approximate solutions of a hierarchical fixed point problem and a variational inequality problem involving a finite family of nonexpansive mappings on a real Hilbert space. Further, we prove that the sequence generated by the proposed iterative method converges to a solution of the hierarchical fixed point problem for a finite family of nonexpansive mappings which is the unique solution of the variational inequality problem. The results presented in this paper are the extension and generalization of some previously known results in this area. An example which satisfies all the conditions of the iterative method and the convergence result is given.
\end{abstract}

MSC: 49J30; 49J40; 47H09; 47J20

Keywords: nonexpansive mapping; strongly monotone; variational inequalities; fixed point problem

\section{Introduction}

Throughout this paper, we always assume that $\mathcal{V}$ is a real Hilbert space with the inner product $\langle\cdot, \cdot\rangle$ and the norm $\|\cdot\|$, respectively. Let a nonlinear mapping $S: \mathcal{V} \rightarrow \mathcal{V}$ be a nonexpansive operator if

$$
\|S u-S v\| \leq\|u-v\|, \quad \forall u, v \in \mathcal{V} .
$$

A point $u \in \mathcal{V}$ is said to be a fixed point of $S$ provided $S u=u$. In this paper, we use $\bar{F}(S)$ to denote the fixed point set which is closed and convex, see [1].

Let $S: W \rightarrow \mathcal{V}$ be a nonexpansive mapping, where $W$ is a nonempty closed convex subset of $\mathcal{V}$. The hierarchical fixed point problem (in short, HFPP) is to find $u \in \bar{F}(S)$ such that

$$
\langle u-S u, v-u\rangle \geq 0, \quad \forall v \in \bar{F}(S) .
$$

Many authors solve (1.1) by various methods, see [2-9] and the references therein.

(c) The Author(s) 2017. This article is distributed under the terms of the Creative Commons Attribution 4.0 International License (http://creativecommons.org/licenses/by/4.0/), which permits unrestricted use, distribution, and reproduction in any medium, provided you give appropriate credit to the original author(s) and the source, provide a link to the Creative Commons license, and indicate if changes were made. 
Yao et al. [2] proposed the following iterative algorithm to solve HFPP (1.1):

$$
\left\{\begin{array}{l}
v_{n}=b_{n} S u_{n}+\left(1-b_{n}\right) u_{n}, \\
u_{n+1}=P_{W}\left[a_{n} g\left(u_{n}\right)+\left(1-a_{n}\right) S v_{n}\right], \quad \forall n \geq 0,
\end{array}\right.
$$

where $\left\{a_{n}\right\}$ and $\left\{b_{n}\right\}$ are sequences in $(0,1)$ and $g: W \rightarrow \mathcal{V}$ is a contraction mapping, and the sequence $\left\{u_{n}\right\}$ generated by (1.2) converges strongly to $z \in \bar{F}(S)$, which is also a unique solution of the variational inequality problem (VIP), i.e., to find $z \in \bar{F}(S)$ such that

$$
\langle(I-g) z, v-z\rangle \geq 0, \quad \forall v \in \bar{F}(S)
$$

After that, Ceng et al. [6] introduced the following algorithm:

$$
u_{n+1}=P_{W}\left[a_{n} \rho g\left(u_{n}\right)+\left(I-a_{n} \mu F\right) S\left(u_{n}\right)\right], \quad \forall n \geq 0,
$$

where $F$ is a Lipschitz continuous and strongly monotone mapping, $g$ is a Lipschitz continuous mapping. Compute an iterative sequence $\left\{u_{n}\right\}$ generated by (1.4) converging strongly to $z \in \bar{F}(S)$, which is also a unique solution of the following variational inequality problem (VIP), i.e., to find $z \in \bar{F}(S)$ such that

$$
\langle\rho g(z)-\mu F(z), v-z| \geq 0, \quad \forall v \in \bar{F}(S) .
$$

By using a $T_{n}$-mapping [10], Yao [11] proposed the following iterative method:

$$
u_{n+1}=a_{n} c g\left(u_{n}\right)+b u_{n}+\left[(1-b) I-a_{n} A\right] T_{n} u_{n}, \quad \forall n \geq 0,
$$

where $c>0, A$ is a strongly positive bounded linear operator and $g: W \rightarrow \mathcal{V}$ is a contraction mapping.

Further, Ceng et al. [12] proposed explicit and implicit iterative schemes for finding a common solution for the set of fixed points of a nonexpansive mapping. Buong and Duong [13] studied the explicit iterative algorithm for finding the approximate solution of a VIP defined over the set of common fixed points of a finite number of nonexpansive mappings:

$$
u_{k+1}=\left(1-b_{k}^{0}\right) u_{k}+b_{k}^{0} S_{0}^{k} S_{p}^{k} \cdots S_{1}^{k} u_{k}
$$

where $S_{i}^{k}=\left(1-b_{k}^{i}\right) u_{k}+b_{k}^{i} S^{i}$ for $1 \leq i \leq p,\left\{S_{i}\right\}_{i=1}^{p}$ are $p$-nonexpansive mappings on a real Hilbert space $\mathcal{V}, S_{0}^{k}=I-\lambda_{k} \mu F$, and $F$ is an $\eta$-strongly monotone and $L$-Lipschitz continuous mapping.

Very recently, Zhang and Yang [14] studied the more general explicit iterative algorithm

$$
u_{k+1}=a_{k} c g\left(u_{k}\right)+\left(I-\mu a_{k} F\right) S_{p}^{k} S_{p-1}^{k} \cdots S_{1}^{k} u_{k}
$$

where $g$ is an $\alpha$-Lipschitzian, $F$ is an $\eta$-strongly monotone and $L$-Lipschitz continuous mapping and $S_{i}^{k}=\left(1-b_{k}^{i}\right) u_{k}+b_{k}^{i} S^{i}$ for $1 \leq i \leq p$. Under some assumptions, compute an 
iterative sequence $\left\{u_{k}\right\}$ proposed by the iterative algorithm (1.8) that strongly converges to the solution of the VIP, i.e., to find $z \in \bigcap_{i=1}^{p} \bar{F}\left(S_{i}\right)$ such that

$$
\langle(\mu F-\gamma g) z, v-z) \geq 0, \quad \forall v \in \bigcap_{i=1}^{p} \bar{F}\left(S_{i}\right) .
$$

Inspired and motivated by the recent research, we develop an iterative algorithm for a hierarchical fixed point problem of a finite family of nonexpansive mappings on the real Hilbert space. We generate a strong convergence theorem for the sequence considered by the generalized method. Numerical examples are also given for the theoretical verification of the algorithm. The algorithm and results presented in this paper improve and extend some recent corresponding algorithms and results; see $[15,16]$ and the references therein.

\section{Preliminaries}

We recall some concepts and results which are needed in the sequel.

Definition 2.1 Let $S: W \rightarrow \mathcal{V}$ be a mapping which is said to be

(i) monotone if

$$
\langle S u-S v, u-v\rangle \geq 0, \quad \forall u, v \in W
$$

(ii) strongly monotone if there exists a constant $\alpha>0$ such that

$$
\langle S u-S v, u-v\rangle \geq \alpha\|u-v\|^{2}, \quad \forall u, v \in W ;
$$

(iii) Lipschitz continuous if there exists a constant $k>0$ such that

$$
\|S u-S v\| \leq k\|u-v\|, \quad \forall u, v \in W .
$$

If $k=1$, then $S$ is called nonexpansive.

Definition 2.2 A mapping $g: W \rightarrow \mathcal{V}$ is said to be $\sigma$-contraction if there exists a constant $\sigma \in(0,1)$ such that

$$
\|g u-g v\| \leq \sigma\|u-v\|, \quad \forall u, v \in W .
$$

Lemma 2.1 ([6]) Let $F: W \rightarrow \mathcal{V}$ be an $\eta$-strongly monotone and $k$-Lipschitz continuous mapping and $g: W \rightarrow \mathcal{V}$ be a $\tau$-Lipschitz continuous mapping. Then the mapping $\mu F-\rho g$ is $(\mu \eta-\rho \tau)$-strongly monotone with condition $\mu \eta>\rho \tau \geq 0$, i.e.,

$$
\langle(\mu F-\rho g) u-(\mu F-\rho g) v, u-v\rangle \geq(\mu \eta-\rho \tau)\|u-v\|^{2}, \quad \forall u, v \in W .
$$

Definition 2.3 A mapping $T: \mathcal{V} \rightarrow \mathcal{V}$ is said to be an averaged mapping if it can be written as the average of the identity $I$ and a nonexpansive mapping, i.e.,

$$
T \equiv(1-\alpha) I+\alpha S
$$

where $\alpha \in(0,1)$ and $S: \mathcal{V} \rightarrow \mathcal{V}$ is nonexpansive. 
Lemma $2.2([17,18])$ If the mappings $\left\{S_{i}\right\}_{i=1}^{p}$ are averaged and have a common fixed point, then

$$
\bigcap_{i=1}^{p} \bar{F}\left(S_{i}\right)=\bar{F}\left(S_{1} S_{2} \cdots S_{p}\right) .
$$

In particular, if $p=2$, we have $\bar{F}\left(S_{1}\right) \cap \bar{F}\left(S_{2}\right)=\bar{F}\left(S_{1} S_{2}\right)=\bar{F}\left(S_{2} S_{1}\right)$.

Lemma 2.3 ([19]) Let $\left\{\alpha_{n}\right\}$ be a sequence of nonnegative real numbers such that

$$
\alpha_{n+1} \leq\left(1-w_{n}\right) \alpha_{n}+t_{n}
$$

where $\left\{w_{n}\right\} \in(0,1)$ and $\left\{t_{n}\right\}$ is a sequence such that

(i) $\sum_{n=1}^{\infty} w_{n}=\infty$;

(ii) $\lim \sup _{n \rightarrow \infty} \frac{t_{n}}{w_{n}} \leq 0$ or $\sum_{n=1}^{\infty}\left|t_{n}\right|<\infty$.

Then $\lim _{n \rightarrow \infty} \alpha_{n}=0$.

Lemma 2.4 ([1]) Let $S: W \rightarrow W$ be a nonexpansive mapping with $\bar{F}(S) \neq \varnothing$. Then the mapping $I-S$ is demiclosed at 0 , that is, if $\left\{u_{n}\right\}$ is a sequence converging weakly to $u$ and $\left\{(I-S) u_{n}\right\}$ converges strongly to 0 , then $(I-S) u=0$.

Lemma 2.5 ([20]) Let $F: W \rightarrow \mathcal{V}$ be an $\eta$-strongly monotone and $k$-Lipschitzian mapping. Let $\frac{2 \eta}{k^{2}}>\mu>0, Q=I-\lambda \mu F$. Then $Q$ is a $(1-\lambda \tau)$-contraction mapping with $\min \left\{1, \frac{1}{\tau}\right\}>\lambda>$ 0 , that is,

$$
\|Q u-Q v\| \leq(1-\lambda \tau)\|u-v\|, \quad \forall u, v \in W
$$

where $\tau=1-\sqrt{1-\mu\left(2 \eta-\mu k^{2}\right)} \in(0,1]$.

Lemma 2.6 Let $\mathcal{V}$ be a real Hilbert space. The following inequality holds:

$$
\|u+v\|^{2} \leq\|u\|^{2}+2\langle v, u+v\rangle, \quad \forall u, v \in \mathcal{V} .
$$

\section{Main results}

In this section, we establish an iterative method for finding the solution of hierarchical fixed point problem (1.1).

Let $W$ be a nonempty closed convex subset of a real Hilbert space $\mathcal{V}$, and let $\left\{S_{i}\right\}_{i=1}^{p}$ be $p$ nonexpansive mappings on $W$ such that $\Xi=\bigcap_{i=1}^{p} \bar{F}\left(S_{i}\right) \neq \emptyset$. Let $F: W \rightarrow W$ be an $\eta$-strongly monotone and $k$-Lipschitzian mapping and $g: W \rightarrow W$ be a $\tau$-contraction mapping.

We consider the following hierarchical fixed point problem (in short, HFPP): find $u \in \Xi$ such that

$$
\langle\rho g(u)-\mu F(u), v-u\rangle \leq 0, \quad \forall v \in \Xi=\bigcap_{i=1}^{p} \bar{F}\left(S_{i}\right) .
$$

Now we define the following algorithm for finding a solution of HFPP (3.1). 
Algorithm 3.1 Given arbitrarily $u_{0} \in W$, compute sequences $\left\{u_{n}\right\}$ and $\left\{v_{n}\right\}$ by the iterative schemes

$$
\left\{\begin{array}{l}
v_{n}=b_{n} u_{n}+\left(1-b_{n}\right) S_{p}^{n} S_{p-1}^{n} \cdots S_{1}^{n} u_{n}, \\
u_{n+1}=a_{n} \rho g\left(v_{n}\right)+c_{n} v_{n}+\left[\left(1-c_{n}\right) I-a_{n} \mu F\right] S_{p}^{n} S_{p-1}^{n} \cdots S_{1}^{n} v_{n}, \quad \forall n \geq 0
\end{array}\right.
$$

where $S_{i}^{n}=\left(1-d_{n}^{i}\right) I+d_{n}^{i} S_{i}$ and $d_{n}^{i} \in(0,1)$ for $i=1,2, \ldots, p$, let the parameters satisfy $\frac{2 \eta}{k^{2}}>$ $\mu>0$ and $\frac{v}{\tau}>\rho>0$, where $v=\mu\left(\eta-\frac{\mu k^{2}}{2}\right)$ and $\left\{a_{n}\right\},\left\{b_{n}\right\}$ and $\left\{c_{n}\right\}$ are sequences in $(0,1)$ satisfying the following conditions:

(i) $\lim _{n \rightarrow \infty} a_{n}=0$ and $\sum_{n=1}^{\infty} a_{n}=\infty$ and $\sum_{n=1}^{\infty}\left|a_{n-1}-a_{n}\right|<\infty$.

(ii) $\left\{b_{n}\right\} \subset[\sigma, 1)$ and $\lim _{n \rightarrow \infty} b_{n}=b<1$.

(iii) $a_{n}+c_{n}<1$ and $\lim _{n \rightarrow \infty} c_{n}=0$.

(iv) $\sum_{n=1}^{\infty}\left|c_{n-1}-c_{n}\right|<\infty$ and $\sum_{n=1}^{\infty}\left|d_{n-1}^{i}-d_{n}^{i}\right|<\infty$ for $i=1,2, \ldots, p$.

Lemma 3.1 Let $u^{*} \in \Xi$. Then the sequences $\left\{u_{n}\right\}$ and $\left\{v_{n}\right\}$ defined in Algorithm 3.1 are bounded.

Proof Let $u^{*} \in \Xi$. So, we have

$$
\begin{aligned}
\left\|v_{n}-u^{*}\right\| & =\left\|b_{n} u_{n}+\left(1-b_{n}\right) S_{p}^{n} S_{p-1}^{n} \cdots S_{1}^{n} u_{n}-u^{*}\right\| \\
& =\left\|\left(1-b_{n}\right)\left(S_{p}^{n} S_{p-1}^{n} \cdots S_{1}^{n} u_{n}-u^{*}\right)+b_{n}\left(u_{n}-u^{*}\right)\right\| \\
& \leq\left(1-b_{n}\right)\left\|u_{n}-u^{*}\right\|+b_{n}\left\|u_{n}-u^{*}\right\| \\
& =\left\|u_{n}-u^{*}\right\| .
\end{aligned}
$$

From (3.2) and (3.3), we have

$$
\begin{aligned}
\left\|u_{n+1}-u^{*}\right\|= & \left\|a_{n} \rho g\left(v_{n}\right)+c_{n} v_{n}+\left[\left(1-c_{n}\right) I-a_{n} \mu F\right] S_{p}^{n} S_{p-1}^{n} \cdots S_{1}^{n} v_{n}-u^{*}\right\| \\
= & \| a_{n}\left(\rho g\left(v_{n}\right)-\mu F\left(u^{*}\right)\right)+c_{n}\left(v_{n}-u^{*}\right) \\
& +\left[\left(1-c_{n}\right) I-a_{n} \mu F\right] S_{p}^{n} S_{p-1}^{n} \cdots S_{1}^{n} v_{n} \\
& -\left[\left(1-c_{n}\right) I-a_{n} \mu F\right] S_{p}^{n} S_{p-1}^{n} \cdots S_{1}^{n} u^{*} \| \\
\leq & a_{n}\left\|\rho g\left(v_{n}\right)-\mu F\left(u^{*}\right)\right\|+c_{n}\left\|v_{n}-u^{*}\right\| \\
& +\|\left[\left(1-c_{n}\right) I-a_{n} \mu F\right] S_{p}^{n} S_{p-1}^{n} \cdots S_{1}^{n} v_{n} \\
& -\left[\left(1-c_{n}\right) I-a_{n} \mu F\right] S_{p}^{n} S_{p-1}^{n} \cdots S_{1}^{n} u^{*} \| \\
= & a_{n}\left\|\rho g\left(v_{n}\right)-\mu F\left(u^{*}\right)\right\|+c_{n}\left\|v_{n}-u^{*}\right\| \\
& +\left(1-c_{n}\right)\left\|\left(I-\frac{a_{n} \mu F}{1-c_{n}}\right) S_{p}^{n} S_{p-1}^{n} \cdots S_{1}^{n} v_{n}-\left(I-\frac{a_{n} \mu F}{1-c_{n}}\right) S_{p}^{n} S_{p-1}^{n} \cdots S_{1}^{n} u^{*}\right\| \\
\leq & \left(1-c_{n}\right)\left(1-\frac{a_{n} v}{1-c_{n}}\right)\left\|v_{n}-u^{*}\right\|+c_{n}\left\|v_{n}-u^{*}\right\|+a_{n}\left\|\rho g\left(v_{n}\right)-\mu F\left(u^{*}\right)\right\| \\
\leq & \left(1-a_{n} v\right)\left\|u_{n}-u^{*}\right\|+a_{n} \rho\left\|g\left(v_{n}\right)-g\left(u^{*}\right)\right\|+a_{n}\left\|\rho g\left(u^{*}\right)-\mu F\left(u^{*}\right)\right\| \\
\leq & \left(1-a_{n} v\right)\left\|u_{n}-u^{*}\right\|+a_{n} \rho \tau\left\|v_{n}-u^{*}\right\|+a_{n}\left\|\rho g\left(u^{*}\right)-\mu F\left(u^{*}\right)\right\| \\
\leq & \left(1-a_{n}(v-\rho \tau)\right)\left\|u_{n}-u^{*}\right\|+a_{n}\left\|\rho g\left(u^{*}\right)-\mu F\left(u^{*}\right)\right\|
\end{aligned}
$$




$$
\begin{aligned}
& \leq\left(1-a_{n}(v-\rho \tau)\right)\left\|u_{n}-u^{*}\right\|+a_{n}(v-\rho \tau) \frac{\left\|\rho g\left(u^{*}\right)-\mu F\left(u^{*}\right)\right\|}{(v-\rho \tau)} \\
& \leq \max \left\{\left\|u_{n}-u^{*}\right\|, \frac{\left\|\rho g\left(u^{*}\right)-\mu F\left(u^{*}\right)\right\|}{v-\rho \tau}\right\},
\end{aligned}
$$

where the third and fifth inequalities follow from (3.3) and the second inequality follows from Lemma 2.5 .

By induction on $n$ and (3.4), we have

$$
\left\|u_{n}-u^{*}\right\| \leq \max \left\{\left\|u_{n}-u^{*}\right\|, \frac{1}{v-\tau \rho}\left\|(\rho g-\mu F) u^{*}\right\|\right\} \quad \text { for } n=1,2, \ldots \text { and } u_{o} \in K
$$

Hence, $\left\{u_{n}\right\}$ is bounded; and consequently, we get $\left\{v_{n}\right\},\left\{S v_{n}\right\},\left\{S_{1} u_{n+1}\right\},\left\|S_{1}^{n} u_{n+1}\right\|$, $\left\|S_{2} S_{1}^{n} u_{n+1}\right\|, \ldots,\left\|S_{p-1}^{n} \cdots S_{1}^{n} u_{n+1}\right\|,\left\|S_{p} S_{p-1}^{n} \cdots S_{1}^{n} u_{n+1}\right\|,\left\|S_{p-1}^{n-1} \cdots S_{1}^{n-1} v_{n}\right\|+\left\|S_{p} S_{p-1}^{n-1} \cdots S_{1}^{n-1} v_{n}\right\|+$ $\left\|S_{p-1}^{n-1} \cdots S_{1}^{n-1} u_{n}\right\|+\left\|S_{p} S_{p-1}^{n-1} \cdots S_{1}^{n-1} u_{n}\right\|$ and $\left\{g\left(v_{n}\right)\right\}$ are bounded.

Lemma 3.2 Let $\left\{u_{n}\right\}$ be a sequence generated by Algorithm 3.1. Then

(i) $\lim _{n \rightarrow \infty}\left\|u_{n+1}-u_{n}\right\|=0$.

(ii) $\lim _{n \rightarrow \infty}\left\|u_{n}-S_{p}^{n} S_{p-1}^{n} \cdots S_{1}^{n} u_{n}\right\|=0$.

Proof From the sequence $\left\{v_{n}\right\}$ defined in Algorithm 3.1, we have

$$
\begin{aligned}
\left\|v_{n}-v_{n-1}\right\|= & \| b_{n} u_{n}+\left(1-b_{n}\right) S_{p}^{n} S_{p-1}^{n} \cdots S_{1}^{n} u_{n} \\
& -b_{n-1} u_{n-1}-\left(1-b_{n-1}\right) S_{p}^{n-1} S_{p-1}^{n-1} \cdots S_{1}^{n-1} u_{n-1} \| \\
= & \|\left(1-b_{n}\right)\left(S_{p}^{n} S_{p-1}^{n} \cdots S_{1}^{n} u_{n}-S_{p}^{n-1} S_{p-1}^{n-1} \cdots S_{1}^{n-1} u_{n-1}\right) \\
& -\left(b_{n}-b_{n-1}\right) S_{p}^{n-1} S_{p-1}^{n-1} \cdots S_{1}^{n-1} u_{n-1} \\
& +b_{n}\left(u_{n}-u_{n-1}\right)-\left(b_{n-1}-b_{n}\right) u_{n-1} \| \\
\leq & \left\|u_{n}-u_{n-1}\right\|+\left|b_{n}-b_{n-1}\right|\left\|S_{p}^{n-1} S_{p-1}^{n-1} \cdots S_{1}^{n-1} u_{n-1}-u_{n-1}\right\| \\
& +\left(1-b_{n}\right)\left\|S_{p}^{n} S_{p-1}^{n} \cdots S_{1}^{n} u_{n}-S_{p}^{n-1} S_{p-1}^{n-1} \cdots S_{1}^{n-1} u_{n-1}\right\| .
\end{aligned}
$$

From the definition of $S_{i}^{n}$ it follows that

$$
\begin{aligned}
\left\|S_{2}^{n} S_{1}^{n} v_{n}-S_{2}^{n-1} S_{1}^{n-1} v_{n}\right\| \leq & \left\|S_{2}^{n} S_{1}^{n} v_{n}-S_{2}^{n} S_{1}^{n-1} v_{n}\right\|+\left\|S_{2}^{n} S_{1}^{n-1} v_{n}-S_{2}^{n-1} S_{1}^{n-1} v_{n}\right\| \\
\leq & \left\|S_{1}^{n} v_{n}-S_{1}^{n-1} v_{n}\right\|+\left\|S_{2}^{n} S_{1}^{n-1} v_{n}-S_{2}^{n-1} S_{1}^{n-1} v_{n}\right\| \\
\leq & \left\|\left(1-d_{n}^{1}\right) v_{n}+d_{n}^{1} S_{1} v_{n}-\left(1-d_{n-1}^{1}\right) v_{n}-d_{n-1}^{1} S_{1} v_{n}\right\| \\
& +\|\left(1-d_{n}^{2}\right) S_{1}^{n-1} v_{n}+d_{n}^{2} S_{2} S_{1}^{n-1} v_{n} \\
& -\left(1-d_{n-1}^{2}\right) S_{1}^{n-1} v_{n}-d_{n-1}^{2} S_{2} S_{1}^{n-1} v_{n} \| \\
\leq & \left|d_{n}^{1}-d_{n-1}^{1}\right|\left(\left\|v_{n}\right\|+\left\|S_{1} v_{n}\right\|\right) \\
& +\left|d_{n}^{2}-d_{n-1}^{2}\right|\left(\left\|S_{1}^{n-1} v_{n}\right\|+\left\|S_{2} S_{1}^{n-1} v_{n}\right\|\right)
\end{aligned}
$$


and from (3.6), we have

$$
\begin{aligned}
&\left\|S_{3}^{n} S_{2}^{n} S_{1}^{n} v_{n}-S_{3}^{n-1} S_{2}^{n-1} S_{1}^{n-1} v_{n}\right\| \\
& \leq\left\|S_{3}^{n} S_{2}^{n} S_{1}^{n} v_{n}-S_{3}^{n} S_{2}^{n-1} S_{1}^{n-1} v_{n}\right\|+\left\|S_{3}^{n} S_{2}^{n-1} S_{1}^{n-1} v_{n}-S_{3}^{n-1} S_{2}^{n-1} S_{1}^{n-1} v_{n}\right\| \\
& \leq\left\|S_{2}^{n} S_{1}^{n} v_{n}-S_{2}^{n-1} S_{1}^{n-1} v_{n}\right\| \\
&+\|\left(1-d_{n}^{3}\right) S_{2}^{n-1} S_{1}^{n-1} v_{n}+d_{n}^{3} S_{3} S_{2}^{n-1} S_{1}^{n-1} v_{n} \\
& \quad-\left(1-d_{n-1}^{3}\right) S_{2}^{n-1} S_{1}^{n-1} v_{n}-d_{n-1}^{3} S_{3} S_{2}^{n-1} S_{1}^{n-1} v_{n} \| \\
& \leq\left|d_{n}^{1}-d_{n-1}^{1}\right|\left(\left\|v_{n}\right\|+\left\|S_{1} v_{n}\right\|\right)+\left|d_{n}^{2}-d_{n-1}^{2}\right|\left(\left\|S_{1}^{n-1} v_{n}\right\|+\left\|S_{2} S_{1}^{n-1} v_{n}\right\|\right) \\
&+\left|d_{n}^{3}-d_{n-1}^{3}\right|\left(\left\|S_{2}^{n-1} S_{1}^{n-1} v_{n}\right\|+\left\|S_{3} S_{2}^{n-1} S_{1}^{n-1} v_{n}\right\|\right)
\end{aligned}
$$

By induction on $p$, it follows that

$$
\begin{aligned}
&\left\|S_{p}^{n} S_{p-1}^{n} \cdots S_{1}^{n} v_{n}-S_{p}^{n-1} S_{p-1}^{n-1} \cdots S_{1}^{n-1} v_{n}\right\| \\
& \leq \leq d_{n}^{1}-d_{n-1}^{1}\left|\left(\left\|v_{n}\right\|+\left\|S_{1} v_{n}\right\|\right)+\right| d_{n}^{2}-d_{n-1}^{2} \mid\left(\left\|S_{1}^{n-1} v_{n}\right\|+\left\|S_{2} S_{1}^{n-1} v_{n}\right\|\right) \\
&+\cdots+\left|d_{n}^{p}-d_{n-1}^{p}\right|\left(\left\|S_{p-1}^{n-1} \cdots S_{1}^{n-1} v_{n}\right\|+\left\|S_{p} S_{p-1}^{n-1} \cdots S_{1}^{n-1} v_{n}\right\|\right)
\end{aligned}
$$

Similarly,

$$
\begin{aligned}
&\left\|S_{p}^{n} S_{p-1}^{n} \cdots S_{1}^{n} u_{n}-S_{p}^{n-1} S_{p-1}^{n-1} \cdots S_{1}^{n-1} u_{n}\right\| \\
& \leq \mid d_{n}^{1}-d_{n-1}^{1}\left|\left(\left\|u_{n}\right\|+\left\|S_{1} u_{n}\right\|\right)+\right| d_{n}^{2}-d_{n-1}^{2} \mid\left(\left\|S_{1}^{n-1} u_{n}\right\|+\left\|S_{2} S_{1}^{n-1} u_{n}\right\|\right) \\
&+\cdots+\left|d_{n}^{p}-d_{n-1}^{p}\right|\left(\left\|S_{p-1}^{n-1} \cdots S_{1}^{n-1} u_{n}\right\|+\left\|S_{p} S_{p-1}^{n-1} \cdots S_{1}^{n-1} u_{n}\right\|\right) .
\end{aligned}
$$

From (3.5), (3.8) and (3.9), it follows that

$$
\begin{aligned}
\left\|u_{n+1}-u_{n}\right\|= & \| a_{n} \rho g\left(v_{n}\right)+c_{n} v_{n}+\left[\left(1-c_{n}\right) I-a_{n} \mu F\right] S_{p}^{n} S_{p-1}^{n} \cdots S_{1}^{n} v_{n} \\
& -a_{n-1} \rho g\left(v_{n-1}\right)-c_{n-1} v_{n-1} \\
& -\left[\left(1-c_{n-1}\right) I-a_{n-1} \mu F\right] S_{p}^{n-1} S_{p-1}^{n-1} \cdots S_{1}^{n-1} v_{n-1} \| \\
= & \| a_{n} \rho\left(g\left(v_{n}\right)-g\left(v_{n-1}\right)\right)+a_{n} \rho g\left(v_{n-1}\right)-a_{n-1} \rho g\left(v_{n-1}\right) \\
& +c_{n}\left(v_{n}-v_{n-1}\right)+c_{n} v_{n-1}-c_{n-1} v_{n-1} \\
& +\left(\left[\left(1-c_{n}\right) I-a_{n} \mu F\right] S_{p}^{n} S_{p-1}^{n} \cdots S_{1}^{n} v_{n}\right. \\
& \left.-\left[\left(1-c_{n}\right) I-a_{n} \mu F\right] S_{p}^{n-1} S_{p-1}^{n-1} \cdots S_{1}^{n-1} v_{n-1}\right) \\
& +\left[\left(1-c_{n}\right) I-a_{n} \mu F\right] S_{p}^{n-1} S_{p-1}^{n-1} \cdots S_{1}^{n-1} v_{n-1} \\
& -\left[\left(1-c_{n-1}\right) I-a_{n-1} \mu F\right] S_{p}^{n-1} S_{p-1}^{n-1} \cdots S_{1}^{n-1} v_{n-1} \| \\
= & \| a_{n} \rho\left(g\left(v_{n}\right)-g\left(v_{n-1}\right)\right)+\left(a_{n}-a_{n-1}\right) \rho g\left(v_{n-1}\right) \\
& +c_{n}\left(v_{n}-v_{n-1}\right)+\left(c_{n}-c_{n-1}\right) v_{n-1} \\
& +\left(\left[\left(1-c_{n}\right) I-a_{n} \mu F\right]\left(S_{p}^{n} S_{p-1}^{n} \cdots S_{1}^{n} v_{n}-S_{p}^{n-1} S_{p-1}^{n-1} \cdots S_{1}^{n-1} v_{n-1}\right)\right) \\
& +\left(\left[\left(1-c_{n}\right) I-a_{n} \mu F\right]-\left[\left(1-c_{n-1}\right) I-a_{n-1} \mu F\right]\right) S_{p}^{n-1} S_{p-1}^{n-1} \cdots S_{1}^{n-1} v_{n-1} \|
\end{aligned}
$$




$$
\begin{aligned}
& \leq a_{n} \rho \tau\left\|v_{n}-v_{n-1}\right\|+\left|a_{n}-a_{n-1}\right|\left\|\rho g\left(v_{n-1}\right)\right\| \\
& +c_{n}\left\|v_{n}-v_{n-1}\right\|+\left|c_{n}-c_{n-1}\right|\left\|v_{n-1}\right\| \\
& +\left(1-c_{n}\right)\left(1-\frac{a_{n} v}{1-c_{n}}\right)\left\|S_{p}^{n} S_{p-1}^{n} \cdots S_{1}^{n} v_{n}-S_{p}^{n-1} S_{p-1}^{n-1} \cdots S_{1}^{n-1} v_{n}\right\| \\
& +\left(\left|c_{n}-c_{n-1}\right|+\left|a_{n}-a_{n-1}\right| \mu F\right)\left\|S_{p}^{n-1} S_{p-1}^{n-1} \cdots S_{1}^{n-1} v_{n-1}\right\| \\
& \leq\left(a_{n} \rho \tau+c_{n}\right)\left\|v_{n}-v_{n-1}\right\| \\
& +\left|a_{n}-a_{n-1}\right|\left(\left\|\rho g\left(v_{n-1}\right)\right\|+\mu F\left\|S_{p}^{n-1} S_{p-1}^{n-1} \cdots S_{1}^{n-1} v_{n-1}\right\|\right) \\
& +\left|c_{n}-c_{n-1}\right|\left(\left\|v_{n-1}\right\|+\left\|S_{p}^{n-1} S_{p-1}^{n-1} \cdots S_{1}^{n-1} v_{n-1}\right\|\right) \\
& +\left(1-c_{n}\right)\left(1-\frac{a_{n} v}{1-c_{n}}\right)\left\|S_{p}^{n} S_{p-1}^{n} \cdots S_{1}^{n} v_{n}-S_{p}^{n-1} S_{p-1}^{n-1} \cdots S_{1}^{n-1} v_{n}\right\| \\
& \leq\left(1-a_{n}(1-\rho \tau)\right)\left\|u_{n}-u_{n-1}\right\| \\
& +\left|a_{n}-a_{n-1}\right|\left(\left\|\rho g\left(v_{n-1}\right)\right\|+\mu F\left\|S_{p}^{n-1} S_{p-1}^{n-1} \cdots S_{1}^{n-1} v_{n-1}\right\|\right) \\
& +\left|b_{n}-b_{n-1}\right|\left\|S_{p}^{n-1} S_{p-1}^{n-1} \cdots S_{1}^{n-1} u_{n-1}-u_{n-1}\right\| \\
& +\left|c_{n}-c_{n-1}\right|\left(\left\|v_{n-1}\right\|+\left\|S_{p}^{n-1} S_{p-1}^{n-1} \cdots S_{1}^{n-1} v_{n-1}\right\|\right) \\
& +\left(1-c_{n}\right)\left(1-\frac{a_{n} v}{1-c_{n}}\right)\left\|S_{p}^{n} S_{p-1}^{n} \cdots S_{1}^{n} v_{n}-S_{p}^{n-1} S_{p-1}^{n-1} \cdots S_{1}^{n-1} v_{n}\right\| \\
& +\left(1-b_{n}\right)\left\|S_{p}^{n} S_{p-1}^{n} \cdots S_{1}^{n} u_{n}-S_{p}^{n-1} S_{p-1}^{n-1} \cdots S_{1}^{n-1} u_{n}\right\| \\
& \leq\left(1-a_{n}(1-\rho \tau)\right)\left\|u_{n}-u_{n-1}\right\| \\
& +\left|a_{n}-a_{n-1}\right|\left(\left\|\rho g\left(v_{n-1}\right)\right\|+\mu F\left\|S_{p}^{n-1} S_{p-1}^{n-1} \cdots S_{1}^{n-1} v_{n-1}\right\|\right) \\
& +\left|b_{n}-b_{n-1}\right|\left\|S_{p}^{n-1} S_{p-1}^{n-1} \cdots S_{1}^{n-1} u_{n-1}-u_{n-1}\right\| \\
& +\left|c_{n}-c_{n-1}\right|\left(\left\|v_{n-1}\right\|+\left\|S_{p}^{n-1} S_{p-1}^{n-1} \cdots S_{1}^{n-1} v_{n-1}\right\|\right) \\
& +\left|d_{n}^{1}-d_{n-1}^{1}\right|\left(\left\|v_{n}\right\|+\left\|S_{1} v_{n}\right\|+\left\|u_{n}\right\|+\left\|S_{1} u_{n}\right\|\right) \\
& +\left|d_{n}^{2}-d_{n-1}^{2}\right|\left(\left\|S_{1}^{n-1} v_{n}\right\|+\left\|S_{2} S_{1}^{n-1} v_{n}\right\|+\left\|S_{1}^{n-1} u_{n}\right\|+\left\|S_{2} S_{1}^{n-1} u_{n}\right\|\right) \\
& +\cdots+\left|d_{n}^{p}-d_{n-1}^{p}\right|\left(\left\|S_{p-1}^{n-1} \cdots S_{1}^{n-1} v_{n}\right\|+\left\|S_{p} S_{p-1}^{n-1} \cdots S_{1}^{n-1} v_{n}\right\|\right. \\
& \left.+\left\|S_{p-1}^{n-1} \cdots S_{1}^{n-1} u_{n}\right\|+\left\|S_{p} S_{p-1}^{n-1} \cdots S_{1}^{n-1} u_{n}\right\|\right) \\
& \leq\left(1-a_{n}(1-\rho \tau)\right)\left\|u_{n}-u_{n-1}\right\| \\
& +\left|a_{n}-a_{n-1}\right|\left(\left\|\rho g\left(v_{n-1}\right)\right\|+\mu F\left\|S_{p}^{n-1} S_{p-1}^{n-1} \cdots S_{1}^{n-1} v_{n-1}\right\|\right. \\
& \left.+\left\|S_{p}^{n-1} S_{p-1}^{n-1} \cdots S_{1}^{n-1} u_{n-1}-u_{n-1}\right\|\right) \\
& +\left|c_{n}-c_{n-1}\right|\left(\left\|v_{n-1}\right\|+\left\|S_{p}^{n-1} S_{p-1}^{n-1} \cdots S_{1}^{n-1} v_{n-1}\right\|\right) \\
& +\left|d_{n}^{1}-d_{n-1}^{1}\right|\left(\left\|v_{n}\right\|+\left\|S_{1} v_{n}\right\|+\left\|u_{n}\right\|+\left\|S_{1} u_{n}\right\|\right) \\
& +\left|d_{n}^{2}-d_{n-1}^{2}\right|\left(\left\|S_{1}^{n-1} v_{n}\right\|+\left\|S_{2} S_{1}^{n-1} v_{n}\right\|+\left\|S_{1}^{n-1} u_{n}\right\|+\left\|S_{2} S_{1}^{n-1} u_{n}\right\|\right) \\
& +\cdots+\left|d_{n}^{p}-d_{n-1}^{p}\right|\left(\left\|S_{p-1}^{n-1} \cdots S_{1}^{n-1} v_{n}\right\|+\left\|S_{p} S_{p-1}^{n-1} \cdots S_{1}^{n-1} v_{n}\right\|\right. \\
& \left.+\left\|S_{p-1}^{n-1} \cdots S_{1}^{n-1} u_{n}\right\|+\left\|S_{p} S_{p-1}^{n-1} \cdots S_{1}^{n-1} u_{n}\right\|\right) \\
& \leq\left(1-a_{n}(1-\rho \tau)\right)\left\|u_{n}-u_{n-1}\right\|
\end{aligned}
$$




$$
\begin{aligned}
& +M\left(\left|a_{n}-a_{n-1}\right|+\left|c_{n}-c_{n-1}\right|+\left|d_{n}^{1}-d_{n-1}^{1}\right|\right. \\
& \left.+\left|d_{n}^{2}-d_{n-1}^{2}\right|+\cdots+\left|d_{n}^{p}-d_{n-1}^{p}\right|\right),
\end{aligned}
$$

where

$$
\begin{aligned}
M= & \max \left\{\operatorname { s u p } _ { n \geq 1 } \left(\left\|\rho g\left(v_{n-1}\right)\right\|+\mu F\left\|S_{p}^{n-1} S_{p-1}^{n-1} \cdots S_{1}^{n-1} v_{n-1}\right\|\right.\right. \\
& \left.+\left\|S_{p}^{n-1} S_{p-1}^{n-1} \cdots S_{1}^{n-1} u_{n-1}-u_{n-1}\right\|\right), \\
& \sup _{n \geq 1}\left(\left\|S_{p}^{n-1} S_{p-1}^{n-1} \cdots S_{1}^{n-1} v_{n-1}\right\|+\left\|v_{n-1}\right\|\right), \\
& \sup _{n \geq 1}\left(\left\|v_{n}\right\|+\left\|S_{1} v_{n}\right\|+\left\|u_{n}\right\|+\left\|S_{1} u_{n}\right\|\right), \\
& \sup _{n \geq 1}\left(\left\|S_{1}^{n-1} v_{n}\right\|+\left\|S_{2} S_{1}^{n-1} v_{n}\right\|+\left\|S_{1}^{n-1} u_{n}\right\|+\left\|S_{2} S_{1}^{n-1} u_{n}\right\|\right), \\
& \sup _{n \geq 1}\left(\left\|S_{p-1}^{n-1} \cdots S_{1}^{n-1} v_{n}\right\|+\left\|S_{p} S_{p-1}^{n-1} \cdots S_{1}^{n-1} v_{n}\right\|\right. \\
& \left.\left.+\left\|S_{p-1}^{n-1} \cdots S_{1}^{n-1} u_{n}\right\|+\left\|S_{p} S_{p-1}^{n-1} \cdots S_{1}^{n-1} u_{n}\right\|\right)\right\} .
\end{aligned}
$$

From conditions (i) and (iv) of Algorithm 3.1 and Lemma 2.3, we have

$$
\lim _{n \rightarrow \infty}\left\|u_{n+1}-u_{n}\right\|=0
$$

From (3.2), we have

$$
\begin{aligned}
\left\|u_{n}-S_{p}^{n} S_{p-1}^{n} \cdots S_{1}^{n} u_{n}\right\| \leq & \left\|u_{n}-u_{n+1}\right\|+\left\|u_{n+1}-S_{p}^{n} S_{p-1}^{n} \cdots S_{1}^{n} u_{n}\right\| \\
\leq & \left\|u_{n}-u_{n+1}\right\|+\| a_{n} \rho g\left(v_{n}\right)+c_{n} v_{n} \\
& +\left[\left(1-c_{n}\right) I-a_{n} \mu F\right] S_{p}^{n} S_{p-1}^{n} \cdots S_{1}^{n} v_{n}-S_{p}^{n} S_{p-1}^{n} \cdots S_{1}^{n} u_{n} \| \\
\leq & \left\|u_{n}-u_{n+1}\right\|+a_{n}\left\|\rho g\left(v_{n}\right)-\mu F S_{p}^{n} S_{p-1}^{n} \cdots S_{1}^{n} v_{n}\right\| \\
& +c_{n}\left\|v_{n}-S_{p}^{n} S_{p-1}^{n} \cdots S_{1}^{n} v_{n}\right\| \\
& +\left\|S_{p}^{n} S_{p-1}^{n} \cdots S_{1}^{n} v_{n}-S_{p}^{n} S_{p-1}^{n} \cdots S_{1}^{n} u_{n}\right\| \\
\leq & \left\|u_{n}-u_{n+1}\right\|+a_{n}\left\|\rho g\left(v_{n}\right)-\mu F S_{p}^{n} S_{p-1}^{n} \cdots S_{1}^{n} v_{n}\right\| \\
& +c_{n}\left\|v_{n}-S_{p}^{n} S_{p-1}^{n} \cdots S_{1}^{n} v_{n}\right\|+\left\|v_{n}-u_{n}\right\| \\
\leq & \left\|u_{n}-u_{n+1}\right\|+a_{n}\left\|\rho g\left(v_{n}\right)-\mu F S_{p}^{n} S_{p-1}^{n} \cdots S_{1}^{n} v_{n}\right\| \\
& +c_{n}\left\|v_{n}-S_{p}^{n} S_{p-1}^{n} \cdots S_{1}^{n} v_{n}\right\| \\
& +\left\|b_{n} u_{n}+\left(1-b_{n}\right) S_{p}^{n} S_{p-1}^{n} \cdots S_{1}^{n} u_{n}-u_{n}\right\| \\
\leq & \left\|u_{n}-u_{n+1}\right\|+a_{n}\left\|\rho g\left(v_{n}\right)-\mu F S_{p}^{n} S_{p-1}^{n} \cdots S_{1}^{n} v_{n}\right\| \\
& +c_{n}\left\|v_{n}-S_{p}^{n} S_{p-1}^{n} \cdots S_{1}^{n} v_{n}\right\|+\left(1-b_{n}\right)\left\|S_{p}^{n} S_{p-1}^{n} \cdots S_{1}^{n} u_{n}-u_{n}\right\| .
\end{aligned}
$$


From (3.11), we have

$$
\begin{aligned}
b_{n}\left\|S_{p}^{n} S_{p-1}^{n} \cdots S_{1}^{n} u_{n}-u_{n}\right\| \leq & \left\|u_{n}-u_{n+1}\right\|+a_{n}\left\|\rho g\left(v_{n}\right)-\mu F S_{p}^{n} S_{p-1}^{n} \cdots S_{1}^{n} v_{n}\right\| \\
& +c_{n}\left\|v_{n}-S_{p}^{n} S_{p-1}^{n} \cdots S_{1}^{n} v_{n}\right\| .
\end{aligned}
$$

Since from (i), (ii), (iii) and (3.10), we have

$$
\lim _{n \rightarrow \infty}\left\|u_{n}-S_{p}^{n} S_{p-1}^{n} \cdots S_{1}^{n} u_{n}\right\|=0
$$

Lemma 3.3 Let

$$
u_{n}=a_{n} \rho g\left(u_{n}\right)+c_{n} u_{n}+\left[\left(1-c_{n}\right) I-a_{n} \mu F\right] S_{p}^{n} S_{p-1}^{n} \cdots S_{1}^{n} u_{n}
$$

Then $u_{n}$ converges strongly to $\tilde{u} \in \Xi$ as $n \rightarrow 0$.

Proof Since $\left\{u_{n}\right\}$ is bounded, we assume that $\left\{u_{n}\right\}$ converges weakly to a point $\tilde{u} \in W$. From Lemma 2.4, we have $\tilde{u} \in \Xi$. Now, for $\tilde{u} \in \Xi$, we get

$$
\begin{aligned}
\left\|u_{n}-\tilde{u}\right\|^{2}= & \left\|a_{n} \rho g\left(u_{n}\right)+c_{n} u_{n}+\left[\left(1-c_{n}\right) I-a_{n} \mu F\right] S_{p}^{n} S_{p-1}^{n} \cdots S_{1}^{n} u_{n}-\tilde{u}\right\|^{2} \\
\leq & \left\langle a_{n}\left(\rho g\left(u_{n}\right)-\mu F(\tilde{u})\right)+c_{n}\left(u_{n}-\tilde{u}\right)\right. \\
& \left.+\left[\left(1-c_{n}\right) I-a_{n} \mu F\right]\left(S_{p}^{n} S_{p-1}^{n} \cdots S_{1}^{n} u_{n}-S_{p}^{n} S_{p-1}^{n} \cdots S_{1}^{n} \tilde{u}\right), u_{n}-\tilde{u}\right\rangle \\
= & \left\langle a_{n} \rho\left(g\left(u_{n}\right)-g(\tilde{u})\right), u_{n}-\tilde{u}\right\rangle \\
& +a_{n}\left\langle\rho g(\tilde{u})-\mu F(\tilde{u}), u_{n}-\tilde{u}\right\rangle+c_{n}\left\langle u_{n}-\tilde{u}, u_{n}-\tilde{u}\right\rangle \\
& +\left[\left(1-c_{n}\right) I-a_{n} \mu F\right]\left\langle S_{p}^{n} S_{p-1}^{n} \cdots S_{1}^{n} u_{n}-S_{p}^{n} S_{p-1}^{n} \cdots S_{1}^{n} \tilde{u}, u_{n}-\tilde{u}\right\rangle \\
\leq & a_{n} \rho \tau\left\|u_{n}-\tilde{u}\right\|^{2}+a_{n}\left\langle\rho g(\tilde{u})-\mu F(\tilde{u}), u_{n}-\tilde{u}\right\rangle \\
& +c_{n}\left\|u_{n}-\tilde{u}\right\|^{2}+\left[\left(1-c_{n}\right) I-a_{n} \mu F\right]\left\|u_{n}-\tilde{u}\right\|^{2} \\
\leq & \left(1-a_{n}(\mu F-\rho \tau)\right)\left\|u_{n}-\tilde{u}\right\|^{2}+a_{n}\left\langle\rho g(\tilde{u})-\mu F(\tilde{u}), u_{n}-\tilde{u}\right\rangle .
\end{aligned}
$$

Hence,

$$
\left\|u_{n}-\tilde{u}\right\|^{2} \leq \frac{1}{(\mu F-\rho \tau)}\left\langle\rho g(\tilde{u})-\mu F(\tilde{u}), u_{n}-\tilde{u}\right\rangle
$$

Since $u_{n} \rightarrow \tilde{u}$, from (3.13) we obtain $u_{n} \rightarrow \tilde{u}$.

Theorem 3.1 The sequence $\left\{u_{n}\right\}$ generated by Algorithm 3.1 converges strongly to $z \in \Xi=$ $\bigcap_{i=1}^{p} \bar{F}\left(S_{i}\right)$, which is also a unique solution of the HFPP

$$
\langle\rho g(z)-\mu F(z), u-z\rangle \leq 0, \quad \forall u \in \Xi
$$

Proof Let $u_{t} \in W$ be a unique fixed point. Now, we claim that

$$
\lim _{n \rightarrow \infty} \sup \left\langle\rho g(z)-\mu F(z), z-u_{n}\right\rangle \leq 0
$$

where $z=\lim _{t \rightarrow 0} u_{t}$. It follows from Lemma 3.3 that $z \in \Xi$. 
By using Lemma 2.6, we get

$$
\begin{aligned}
\left\|u_{n}-u_{t}\right\|^{2}= & \left\|a_{n} \rho g\left(u_{n}\right)+c_{n} u_{n}+\left[\left(1-c_{n}\right) I-a_{n} \mu F\right] S_{p}^{n} S_{p-1}^{n} \cdots S_{1}^{n} u_{n}-u_{t}\right\|^{2} \\
= & \| a_{n}\left(\rho g\left(u_{n}\right)-\mu F\left(u_{t}\right)\right)+c_{n}\left(u_{n}-u_{t}\right) \\
& +\left[\left(1-c_{n}\right) I-a_{n} \mu F\right] S_{p}^{n} S_{p-1}^{n} \cdots S_{1}^{n} u_{n} \\
& -\left[\left(1-c_{n}\right) I-a_{n} \mu F\right] S_{p}^{n} S_{p-1}^{n} \cdots S_{1}^{n} u_{t} \|^{2} \\
\leq & \| c_{n}\left(u_{n}-u_{t}\right)+\left[\left(1-c_{n}\right) I-a_{n} \mu F\right] S_{p}^{n} S_{p-1}^{n} \cdots S_{1}^{n} u_{n} \\
& -\left[\left(1-c_{n}\right) I-a_{n} \mu F\right] S_{p}^{n} S_{p-1}^{n} \cdots S_{1}^{n} u_{t} \|^{2}+2 a_{n}\left\langle\rho g\left(u_{n}\right)-\mu F\left(u_{t}\right), u_{n}-u_{t}\right\rangle \\
\leq & \left\{c_{n}\left\|u_{n}-u_{t}\right\|+\left(1-c_{n}\right) \|\left(I-\frac{a_{n} \mu F}{1-c_{n}}\right) S_{p}^{n} S_{p-1}^{n} \cdots S_{1}^{n} u_{n}\right. \\
& \left.-\left(I-\frac{a_{n} \mu F}{1-c_{n}}\right) S_{p}^{n} S_{p-1}^{n} \cdots S_{1}^{n} u_{t} \|\right\}^{2} \\
& +2 a_{n} \rho\left\langle g\left(u_{n}\right)-g\left(u_{t}\right), u_{n}-u_{t}\right\rangle+2 a_{n}\left\langle\rho g\left(u_{t}\right)-\mu F\left(u_{t}\right), u_{n}-u_{t}\right\rangle \\
\leq & \left\{c_{n}\left\|u_{n}-u_{t}\right\|+\left(1-c_{n}\right)\left(I-\frac{a_{n} v}{1-c_{n}}\right)\left\|u_{n}-u_{t}\right\|\right\} \\
& +2 a_{n} \rho \tau\left\|u_{n}-u_{t}\right\|\left\|u_{n}-u_{t}\right\|+2 a_{n}\left\langle\rho g\left(u_{t}\right)-\mu F\left(u_{t}\right), u_{n}-u_{t}\right\rangle \\
\leq & \left\{\left(1-c_{n}\left\|u_{n}-u_{t}\right\|+\left(1-c_{n}-a_{n} v\right)\left\|u_{n}-u_{t}\right\|\right\}^{2}+2 a_{n} \rho \tau\left\|u_{n}-u_{t}\right\|^{2}\right. \\
& +2 a_{n}\left\langle\rho g\left(u_{t}\right)-\mu F\left(u_{t}\right), u_{n}-u_{t}\right\rangle \\
\leq & \\
& \\
&
\end{aligned}
$$

From the above we have

$$
\left\langle\rho g\left(u_{t}\right)-\mu F\left(u_{t}\right), u_{t}-u_{n}\right\rangle \leq \frac{\mathcal{A}_{n}(t)}{2 a_{n}}\left\|u_{n}-u_{t}\right\|^{2},
$$

where $\mathcal{A}_{n}(t)=\left[1-\left[\left(1-a_{n} v\right)^{2}+2 a_{n} \rho \tau\right]\right]$.

Further,

$$
\lim _{n \rightarrow \infty} \sup \left\langle\rho g\left(u_{t}\right)-\mu F\left(u_{t}\right), u_{t}-u_{n}\right\rangle \leq \frac{\mathcal{A}_{n}(t)}{2} \mathcal{M}
$$

where $\mathcal{M}>0$ is a constant such that $\mathcal{M} \geq\left\|u_{n}-u_{t}\right\|^{2}$.

Taking the limsup as $t \rightarrow 0$ in (3.14), we get

$$
\lim _{n \rightarrow \infty} \sup \left\langle\rho g(z)-\mu F(z), z-u_{n}\right\rangle \leq 0
$$

Now, we have to show that $u_{n} \rightarrow z$.

$$
\begin{aligned}
\left\|u_{n+1}-z\right\|^{2} & =\left\|a_{n} \rho g\left(v_{n}\right)+c_{n} v_{n}+\left[\left(1-c_{n}\right) I-a_{n} \mu F\right] S_{p}^{n} S_{p-1}^{n} \cdots S_{1}^{n} v_{n}-z\right\|^{2} \\
& =\left\langle a_{n} \rho g\left(v_{n}\right)+c_{n} v_{n}+\left[\left(1-c_{n}\right) I-a_{n} \mu F\right] S_{p}^{n} S_{p-1}^{n} \cdots S_{1}^{n} v_{n}-z, u_{n+1}-z\right\rangle \\
& \leq\left\langle a_{n}\left(\rho g\left(v_{n}\right)-\mu F(z)\right)+c_{n}\left(v_{n}-z\right)+\left(1-c_{n}\right)\left[\left(I-\frac{a_{n} \mu F}{1-c_{n}}\right) S_{p}^{n} S_{p-1}^{n} \cdots S_{1}^{n} v_{n}\right.\right.
\end{aligned}
$$




$$
\begin{aligned}
& \left.\left.-\left(I-\frac{a_{n} \mu F}{1-c_{n}}\right) S_{p}^{n} S_{p-1}^{n} \cdots S_{1}^{n} z\right], u_{n+1}-z\right\rangle \\
= & \left\langle a_{n} \rho\left(g\left(v_{n}\right)-g(z)\right), u_{n+1}-z\right\rangle+a_{n}\left\langle\rho g(z)-\mu F(z), u_{n+1}-z\right\rangle \\
& +c_{n}\left\langle v_{n}-z, u_{n+1}-z\right\rangle+\left(1-c_{n}\right)\left\langle\left(I-\frac{a_{n} \mu F}{1-c_{n}}\right) S_{p}^{n} S_{p-1}^{n} \cdots S_{1}^{n} v_{n}\right. \\
& \left.-\left(I-\frac{a_{n} \mu F}{1-c_{n}}\right) S_{p}^{n} S_{p-1}^{n} \cdots S_{1}^{n} z, u_{n+1}-z\right\rangle \\
\leq & a_{n} \rho \tau\left\|v_{n}-z\right\|\left\|u_{n+1}-z\right\|+a_{n}\left\langle\rho g(z)-\mu F(z), u_{n+1}-z\right\rangle \\
& +c_{n}\left\|v_{n}-z\right\|\left\|u_{n+1}-z\right\|+\left(1-c_{n}-a_{n} v\right)\left\|v_{n}-z\right\|\left\|u_{n+1}-z\right\| \\
\leq & \left(a_{n} \rho \tau+1-a_{n} v\right)\left\|v_{n}-z\right\|\left\|u_{n+1}-z\right\|+a_{n}\left\langle\rho g(z)-\mu F(z), u_{n+1}-z\right\rangle \\
\leq & \left(1-a_{n}(v-\rho \tau)\right)\left\|v_{n}-z\right\|\left\|u_{n+1}-z\right\|+a_{n}\left\langle\rho g(z)-\mu F(z), u_{n+1}-z\right\rangle \\
\leq & \left(1-a_{n}(v-\rho \tau)\right)\left\|u_{n}-z\right\|\left\|u_{n+1}-z\right\|+a_{n}\left\langle\rho g(z)-\mu F(z), u_{n+1}-z\right\rangle \\
\leq & \frac{\left(1-a_{n}(v-\rho \tau)\right)}{2}\left(\left\|u_{n}-z\right\|^{2}+\left\|u_{n+1}-z\right\|^{2}\right) \\
& +a_{n}\left\langle\rho g(z)-\mu F(z), u_{n+1}-z\right\rangle .
\end{aligned}
$$

Further,

$$
\begin{aligned}
& {\left[1-\frac{\left(1-a_{n}(v-\rho \tau)\right)}{2}\right]\left\|u_{n+1}-z\right\|^{2} \leq\left[\frac{1-a_{n}(v-\rho \tau)}{2}\right]\left\|u_{n}-z\right\|^{2}} \\
& +a_{n}\left\langle\rho g(z)-\mu F(z), u_{n+1}-z\right\rangle, \\
& {\left[\frac{1+a_{n}(v-\rho \tau)}{2}\right]\left\|u_{n+1}-z\right\|^{2} \leq\left[\frac{1-a_{n}(v-\rho \tau)}{2}\right]\left\|u_{n}-z\right\|^{2}} \\
& +a_{n}\left\langle\rho g(z)-\mu F(z), u_{n+1}-z\right\rangle \text {, }
\end{aligned}
$$

which implies that

$$
\begin{aligned}
\left\|u_{n+1}-z\right\|^{2} \leq & {\left[\frac{1-a_{n}(v-\rho \tau)}{1+a_{n}(v-\rho \tau)}\right]\left\|u_{n}-z\right\|^{2} } \\
& +\left[\frac{2 a_{n}}{1+a_{n}(v-\rho \tau)}\right]\left\langle\rho g(z)-\mu F(z), u_{n+1}-z\right\rangle, \\
\left\|u_{n+1}-z\right\|^{2} \leq & {\left[1-\frac{2 a_{n}(v-\rho \tau)}{1+a_{n}(v-\rho \tau)}\right]\left\|u_{n}-z\right\|^{2} } \\
& +\left[\frac{2 a_{n}(v-\rho \tau)}{1+a_{n}(v-\rho \tau)}\right]\left\{\frac{1}{v-\rho \tau}\left\langle\rho g(z)-\mu F(z), u_{n+1}-z\right\rangle\right\} .
\end{aligned}
$$

Let $w_{n}=\left[\frac{2 a_{n}(\nu-\rho \tau)}{1+a_{n}(\nu-\rho \tau)}\right]$ and

$$
t_{n}=\left[\frac{2 a_{n}(v-\rho \tau)}{1+a_{n}(v-\rho \tau)}\right]\left\{\frac{1}{v-\rho \tau}\left\langle\rho g(z)-\mu F(z), u_{n+1}-z\right\rangle\right\} .
$$

We have $\sum_{n=1}^{\infty} a_{n}=\infty$ and $\lim _{n \rightarrow \infty} \sup \left\{\frac{1}{v-\rho \tau}\left\langle\rho g(z)-\mu F(z), u_{n+1}-z\right\rangle\right\} \leq 0$. It follows that $\sum_{n=1}^{\infty} w_{n}=\infty$ and $\lim _{n \rightarrow \infty} \sup \frac{t_{n}}{w_{n}} \leq 0$. Thus, all the conditions of Lemma 2.3 are fulfilled. Hence, $u_{n} \rightarrow z$. 


\section{Examples}

The following example ensures that all the conditions of Algorithm 3.1 and the convergence result are fulfilled.

Example 4.1 Let $a_{n}=\frac{1}{3 n}, b_{n}=\frac{2 n-1}{3 n}$ and $c_{n}=\frac{1}{3 n}$. Then

$$
\lim _{n \rightarrow \infty} a_{n}=\frac{1}{3} \lim _{n \rightarrow \infty} \frac{1}{n}=0,
$$

and

$$
\sum_{n=1}^{\infty} a_{n}=\frac{1}{3} \sum_{n=1}^{\infty} \frac{1}{n}=\infty .
$$

The sequence $\left\{a_{n}\right\}$ satisfies condition (i) of Algorithm 3.1.

Now we compute

$$
a_{n-1}-a_{n}=\frac{1}{3(n-1)}-\frac{1}{3 n}=\frac{1}{3}\left(\frac{1}{n-1}-\frac{1}{n}\right)=\frac{1}{3 n(n-1)} .
$$

So,

$$
\sum_{n=1}^{\infty}\left|a_{n-1}-a_{n}\right|<\infty
$$

Similarly, we can show

$$
\sum_{n=1}^{\infty}\left|c_{n-1}-c_{n}\right|<\infty .
$$

The sequences $\left\{a_{n}\right\},\left\{b_{n}\right\}$ and $\left\{c_{n}\right\}$ satisfy conditions (i), (ii) and (iii).

Let $d_{n}^{i}=\frac{n}{n+i}$ for $i=1,2$. Then

$$
\sum_{n=1}^{\infty}\left|d_{n-1}^{i}-d_{n}^{i}\right|<\infty .
$$

Hence the sequence $\left\{d_{n}^{i}\right\}$ also satisfies condition (iv) of Algorithm 3.1.

Let $S_{1}, S_{2}: \mathbb{R} \rightarrow \mathbb{R}$ be defined by

$$
S_{1}(u)=\sin \frac{u}{2}
$$

and

$$
S_{2}(u)=\frac{u}{2}, \quad \forall u \in \mathbb{R},
$$

and let the mapping $g: \mathbb{R} \rightarrow \mathbb{R}$ be defined by

$$
g(u)=\frac{u}{2}+1, \quad \forall u \in \mathbb{R} .
$$

It is easy to verify that $S_{1}$ and $S_{2}$ are $\frac{1}{2}$-nonexpansive and $g$ is a $\frac{1}{2}$-contraction mapping. 
Table 1 The values of $u_{n}$ and $v_{n}$ with the initial values $u_{1}=-10$ and $u_{1}=10$

\begin{tabular}{llllll}
\hline & \multicolumn{1}{l}{$\boldsymbol{u}_{\mathbf{1}}=\mathbf{- 1 0}$} & & \multicolumn{2}{c}{$\boldsymbol{u}_{\mathbf{1}}=\mathbf{1 0}$} & \\
\cline { 2 - 3 } & $\boldsymbol{u}_{\boldsymbol{n}}$ & $\boldsymbol{v}_{\boldsymbol{n}}$ & & $\boldsymbol{u}_{\boldsymbol{n}}$ & $\boldsymbol{v}_{\boldsymbol{n}}$ \\
\hline$n=1$ & -10.0000 & -14.5154 & 10.0000 & 14.6347 \\
$n=2$ & -7.0467 & -7.0467 & 7.8560 & 7.8997 \\
$n=3$ & -2.7768 & -2.7268 & 5.2941 & 5.2981 \\
$n=4$ & -1.0152 & -1.0152 & 2.0321 & 2.0431 \\
$n=5$ & -0.3254 & -0.3252 & 0.8356 & 0.8436 \\
$n=6$ & -0.0703 & -0.0703 & 0.3587 & 0.3487 \\
$n=7$ & -0.0458 & -0.0458 & 0.1662 & 0.1689 \\
$n=8$ & -0.0492 & -0.0429 & 0.0889 & 0.0989 \\
$n=9$ & -0.0399 & -0.0399 & 0.0571 & 0.0671 \\
$n=10$ & -0.0327 & -0.0372 & 0.0429 & 0.0429 \\
$n=11$ & -0.0298 & -0.0331 & 0.0357 & 0.0375 \\
$n=12$ & -0.0297 & -0.0289 & 0.0315 & 0.0351 \\
$n=13$ & -0.0146 & -0.0279 & 0.0286 & 0.0211 \\
$n=14$ & -0.0118 & -0.0164 & 0.0224 & 0.0200 \\
$n=15$ & -0.0109 & -0.0131 & 0.0207 & 0.0198 \\
\hline
\end{tabular}

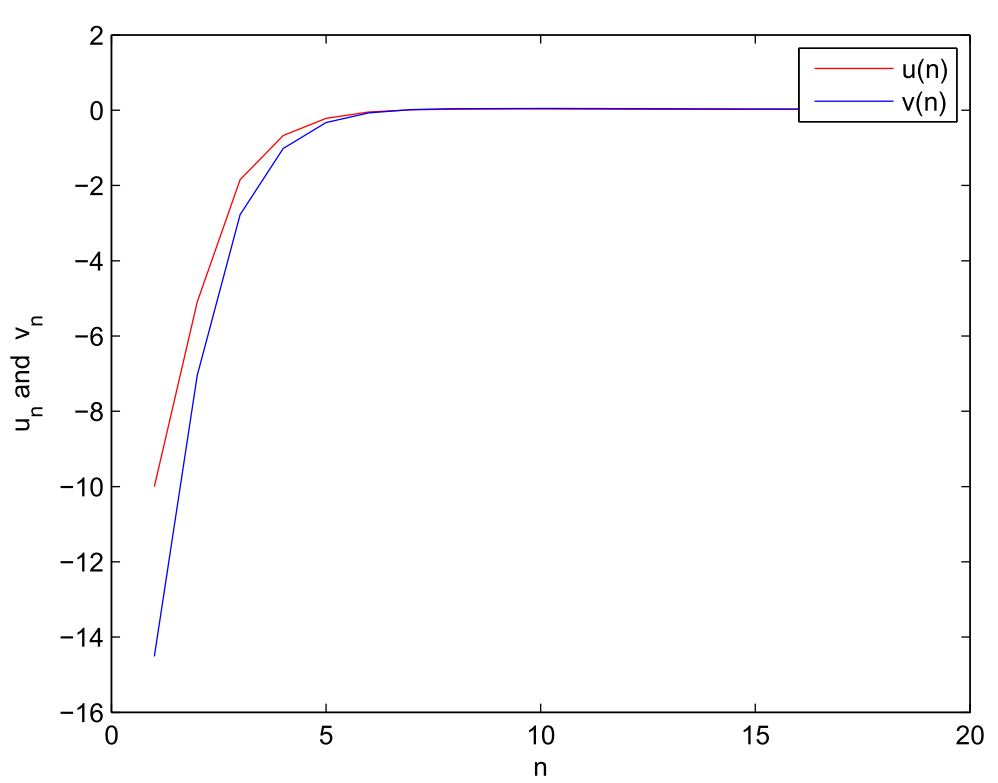

Figure 1 The convergence of $u_{n}$ and $v_{n}$ with the initial value $u_{1}=-10$.

Further,

$$
\Xi=\bigcap_{i=1}^{2} F\left(S_{i}\right)=\{0\} .
$$

Suppose that the mapping $F: \mathbb{R} \rightarrow \mathbb{R}$ is defined by

$$
F(u)=2 u, \quad \forall u \in \mathbb{R} .
$$

Hence, $F$ is 2-strongly monotone and 2-Lipschitzian.

Assume that $\rho=\frac{1}{15}$ and $\mu=\frac{1}{5}$ and they satisfy $0<\mu<\frac{2 \eta}{k^{2}}$ and $0 \leq \rho \tau<\nu$, where $\nu=$ $1-\sqrt{1-\mu\left(2 \eta-\mu k^{2}\right)}$. 


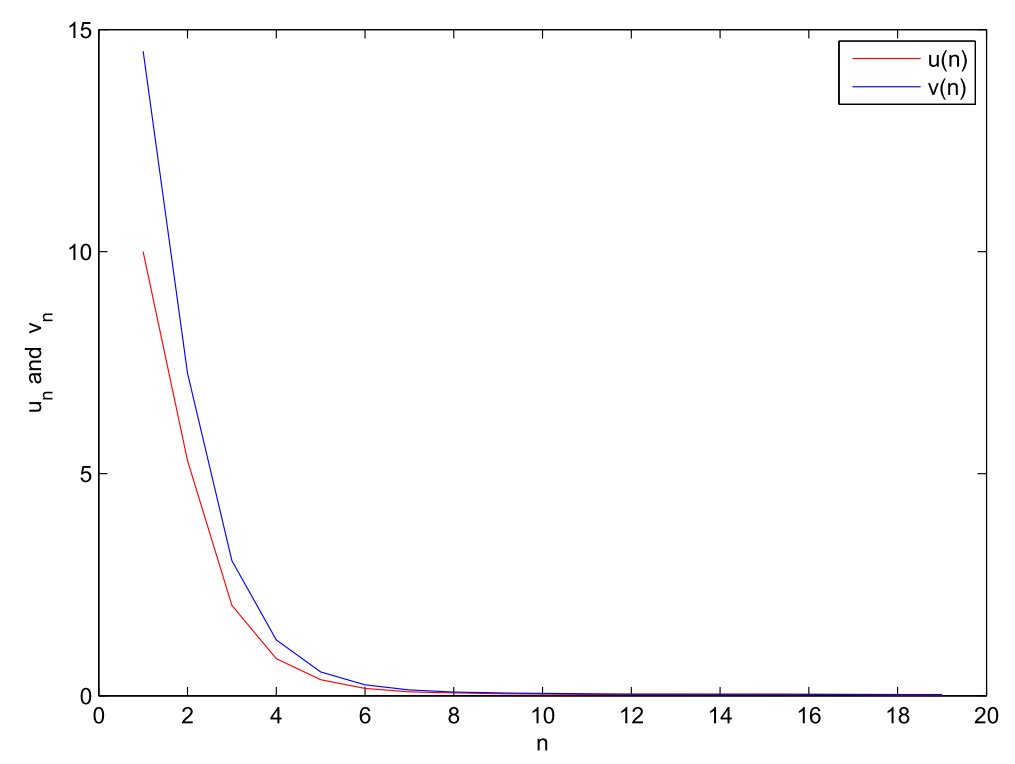

Figure 2 The convergence of $u_{n}$ and $v_{n}$ with the initial value $u_{1}=10$.

All codes were written in Matlab, the values of $\left\{v_{n}\right\}$ and $\left\{u_{n}\right\}$ with different $n$ are given in Table 1.

Remark 4.1 Table 1 and Figures 1 and 2 show that the sequences $\left\{v_{n}\right\}$ and $\left\{u_{n}\right\}$ converge to 0 . Also, $\{0\} \in \Xi$.

\section{Conclusion}

We have analyzed an iterative method for finding an approximate solution of hierarchical fixed point problem (1.1) and variational inequality problem (1.5) involving a finite family of nonexpansive mappings in a real Hilbert space. This method can be viewed as a modification and improvement of some existing methods $[15,16]$ for solving the variational inequality problem and the hierarchical fixed point problem. Therefore, Algorithm 3.1 is expected to be widely applicable.

Competing interests

The authors declare that they have no competing interests.

Authors' contributions

Both the authors contributed equally and approved the final manuscript.

\section{Publisher's Note}

Springer Nature remains neutral with regard to jurisdictional claims in published maps and institutional affiliations.

Received: 20 July 2017 Accepted: 27 October 2017 Published online: 17 November 2017

\section{References}

1. Geobel, K, Kirk, WA: Topics in Metric Fixed Point Theory. Cambridge University Press, Cambridge (1990)

2. Yao, Y, Cho, YJ, Liou, YC: Iterative algorithms for hierarchical fixed points problems and variational inequalities. Math. Comput. Model. 52(9-10), 1697-1705 (2010)

3. Crombez, G: A hierarchical presentation of operators with fixed points on Hilbert spaces. Numer. Funct. Anal. Optim. 27, 259-277 (2006)

4. Moudafi, A: Krasnoselski-Mann iteration for hierarchical fixed-point problems. Inverse Probl. 23(4), 1635-1640 (2007)

5. Gu, G, Wang, S, Cho, YJ: Strong convergence algorithms for hierarchical fixed points problems and variational inequalities. J. Appl. Math. 2011, Article ID 164978 (2011) 
6. Ceng, LC, Ansari, QH, Yao, JC: Iterative methods for hierarchical variational inequalities in Hilbert spaces. J. Optim. Theory Appl. 151, 489-512 (2011)

7. Xu, HK: Viscosity method for hierarchical fixed point approach to variational inequalities. Taiwan. J. Math. 14(2), 463-478 (2010)

8. Wang, $Y, X u, W$ : Strong convergence of a modified iterative algorithm for hierarchical fixed point problems and variational inequalities. Fixed Point Theory Appl. 2013, Article ID 121 (2013)

9. Ansari, QH, Ceng, LC, Gupta, H: Triple hierarchical variational inequalities. In: Ansari, QH (ed.) Nonlinear Analysis: Approximation Theory, Optimization and Applications, pp. 231-280. Springer, New York (2014)

10. Takahashi, W, Atsushiba, S: Strong convergence theorems for a finite family of nonexpansive mappings and applications. Indian J. Math. 41(3), 435-453 (1999)

11. Yao, Y: A general iterative method for a finite family of nonexpansive mappings. Nonlinear Anal. 66, 2676-2678 (2007)

12. Ceng, LC, Khan, AR, Ansari, QH, Yao, JC: Viscosity approximation methods for strongly positive and monotone operators. Fixed Point Theory 10(1), 35-71 (2009)

13. Buong, N, Duong, LT: An explicit iterative algorithm for a class of variational inequalities in Hilbert spaces. J. Optim. Theory Appl. 151, 513-524 (2011)

14. Zhang, C, Yang, C: A new explicit iterative algorithm for solving a class of variational inequalities over the common fixed points set of a finite family of nonexpansive mappings. Fixed Point Theory Appl. 2014, Article ID 60 (2014)

15. Bnouhachem, A, Ansari, QH, Yao, JC: An iterative algorithm for hierarchical fixed point problems for a finite family of nonexpansive mappings. Fixed Point Theory Appl. 2015, Article ID 111 (2015)

16. Bnouhachem, A, Ansari, QH, Yao, JC: Strong convergence algorithm for hierarchical fixed point problems of a finite family of nonexpansive mappings. Fixed Point Theory 17, 47-62 (2016)

17. Byrne, $C:$ A unified treatment of some iterative algorithms in signal processing and image reconstruction. Inverse Probl. 20, 103-120 (2004)

18. Combettes, PL: Solving monotone inclusions via compositions of nonexpansive averaged operators. Optimization 53, 475-504 (2004)

19. Xu, HK: Iterative algorithms for nonlinear operators. J. Lond. Math. Soc. 66, 240-256 (2002)

20. Deng, BC, Chen, T, Li, ZF: Cyclic iterative method for strictly pseudononspreading in Hilbert spaces. J. Appl. Math. 2012, Article ID 435676 (2012)

\section{Submit your manuscript to a SpringerOpen ${ }^{\circ}$ journal and benefit from:}

- Convenient online submission

- Rigorous peer review

- Open access: articles freely available online

- High visibility within the field

- Retaining the copyright to your article

Submit your next manuscript at $\boldsymbol{~ s p r i n g e r o p e n . c o m ~}$ 